\title{
Theory of the Infinite Cylindrical Antenna Including the Feedpoint Singularity in Antenna Current
}

\author{
R. H. Duncan \\ Contribution From Departments of Physics and Electrical Engineering, New Mexico State University, \\ University Park, N. Mex.
}

(Received August 31, 1961; revised October 16, 1961)

\begin{abstract}
The usual idealized model for a cylindrical antenna consists of an extremely thin-walled tube of infinite conductivity with an infinitesimally narrow circumferential gap. Physically, one expects the antenna current at the feedpoint of such a model to be infinite. However, the singularity in feedpoint current is not detected by either iterative or Fourier series solutions of the integral equation for antenna current. These solutions are continuous on $|z| \leq h$, where $h$ is the half-length of the antenna. They are also in good agreement with each other and with experimental data. From a formal point of view this amounts to saying that the conventional solutions of the integral equation are solutions on most, but not all, of the range $|z| \leq h$. Inside a small region, $|z| \leq z_{s}$, the correct solution to the integral equation is peculiar to the idealized generator. Low-order iterative and Fourier series solutions innore these peculiarities and produce a smooth current distribution which can be used in predicting the behavior of practical structures. This paper is concerned with a detailed study of the theoretical current near the feedpoint of the idealized model and the development of a definition of $z_{s}$.
\end{abstract}

\section{Introduction}

The current distribution, $I_{t}(z)$, for a finite cylindrical antenna of length $2 h$ and radius $a$ is a solution of

$$
\frac{\mu}{4 \pi} \int_{-h}^{+h} I_{t}\left(z_{0}\right) g\left(z-z_{\mathrm{o}}\right) d z_{0}=C \cos k z+\frac{\omega \mu \epsilon}{k} \frac{V}{2} e^{-j k|z|}
$$

where

$$
g\left(z-z_{0}\right)=\frac{1}{2 \pi} \int_{-\pi}^{+\pi} \frac{e^{-j k R}}{R} d \phi
$$

and

$$
R=\left[4 a^{2} \sin ^{2} \phi / 2+\left(z-z_{0}\right)^{2}\right]^{\frac{1}{2}} .
$$

Some writers approximate the kernel distance with

$$
R_{1}=\left[a^{2}+\left(z-z_{0}\right)^{2}\right]^{\frac{1}{2}} .
$$

With the correct kernel distance the integral equation corresponds exactly to a model consisting of a thinwalled tube excited by a hypothetical generator of voltage $V$ such that the electric field in a narrow gap is given by

$$
E_{\mathrm{gap}}=-V \delta(z)
$$

Idealization of the generator region leads to a fundamental difficulty. A finite voltage across an infinitesimal gap implies that $I(z)$ is infinite at $z=0$. However, it is possible to manage the solution of (1) in such a way as to obtain a useful finite result.

\footnotetext{
1 This work was carried out under P. O. 14-3270 from Sandia Corporation.
}

the King-Middleton [King, 1956] and Hallén [1938] theories the integral equation is solved by an iterative process in which the starting approximation is a continuous function. After only one or two iterations the output function is still continuous. It can then be argued that these solutions are valid on $|z| \leq h$ except for a small region $|z| \leq z_{s}$ where they differ markedly from the mathematically correct solution. The continuous "solution" evaluated at $z=0$ gives a finite admittance, $Y_{a}$, which is presumed to be characteristic of the antenna viewed as a circuit element. There is still the problem of establishing correspondence with experiment. Measured antenna admittances depend on $Y_{a}$ and the actual terminal zone structure and transmission line used in the experiment. Experimental procedures and theoretical corrections can be designed to extricate $Y_{a}$ from the experimental data [King, 1956]. Combined theoretical and experimental experience have definitely established the utility of approximating the correct solution of (1) by a continuous function.

Iterative solutions of (1) become extremely tedious beyond second or third order. Some of the integrals arising in the theory are performed with the approximate kernel distance given by (4). This approximation is suspect for thick structures. These objections are overcome in a theory developed by Duncan and Hinchey [1960]. This theory provides a method for obtaining the coefficients of a Fourier expansion of the current distribution to any reasonable degree of accuracy. The solution has been carried to 25th order. One interesting result is that no sign of poor behavior of $I(z)$ near $z=0$ develops even at such high order. One does not expect a Fourier series to show 
fine details in a region of width $z_{s}$ until the order of solution is high enough for space frequencies of order $z_{s}^{-1}$ to appear. The fundamental period in finite antenna theory is about a wavelength. Thus, our calculations provide evidence that

$$
z_{s}<\lambda / 25 \text { for } h / a \geq 60,
$$

the smallest $h / a$ ratio studied.

According to Wu and King [1959], $z_{s}$ may be much smaller than the estimate provided by (6). It can be shown that

$$
I(z) \simeq-j \frac{4 k a V}{Z_{0}} \ln k z
$$

for small $z$. If one is allowed to guess that this function is simply not applicable after it decreases to a value comparable to the amplitudes of well-known continuous solutions one obtains

$$
z_{s} \sim \frac{\lambda}{2 \pi} e^{-1 / k a} .
$$

Although (7) is certainly correct for small enough z, the method of obtaining (8) is somewhat arbitrary. A definition of $z_{s}$ based on the point at which a simple logarithmic function exceeds the nominal value of observed current fails to take into account the manner in which the current makes the transition from moderate to logarithmic behavior. The definition of $z_{s}$ to be introduced here is a rough measure of the location of the transition region in which the graph of the current changes from moderate to large slope. On this basis (8) turns out to be much too small and too sensitive to antenna diameter to be useful.

Obviously, the distinction between thin and thick cylinders will always be somewhat arbitrary. Such distinctions depend on methods of analysis and desired numerical tolerances. In this paper a thin antenna is one in which a moderately smooth function is a good approximation to $I(z)$ except within a suitably small fraction of a wavelength near $z=0$. The remainder of the paper is devoted to the quantitative development of this general idea.

In order to facilitate the analysis we allow the antenna length to become infinite. The singularity in $I(z)$ is associated entirely with the idealized generator. Waves reflected from the ends of a finite antenna can do no more than produce a standing wave of twice the amplitude of a smooth approximation to $I(z)$ for the infinite antenna.

A byproduct of this study is the development of a continuous approximation to $I(z)$ for the infinite antenna when $k a$ is small enough to justify such a current distribution. Admittances defined from the approximate solutions for various values of $k a$ are to be interpreted similarly to those of finite antenna theory. Admittance values for infinite antennas are of more than academic interest. Altshuler [1961] recently reported on a traveling-wave linear antenna. Lumped resistors are inserted in this type of antenna at $|z|=h-\lambda / 4$. With appropriate values of resist- ance the current distribution in the central region, $0<|z|<h-\lambda / 4$, consists primarily of running waves traveling outward from $z=0$. It is thus possible to apply the theory of the infinite antenna to a realizable structure.

\section{Formal Theory}

The right-hand side of (1) represents the surface value of vector potential. When the antenna length becomes infinite the term $C \cos k z$ which represents standing waves is no longer needed. With $V=1$ the integral equation becomes

$$
\frac{\mu}{4 \pi} \int_{-\infty}^{+\infty} I_{t}\left(z_{0}\right) g\left(z-z_{0}\right) d z_{0}=\frac{\omega \mu \epsilon}{2 k} e^{-j k|z|} .
$$

Hallén [1956] has reported the solution of (9). In the notation of this paper,

$$
I_{t}(z)=\frac{4 k}{\pi Z_{0}} \int_{0}^{\infty} \frac{\cos \alpha z}{\beta^{2} J_{0}(\beta a) H_{0}^{(2)}(\beta a)} d \alpha,
$$

where $\beta^{2}=k^{2}-\alpha^{2}$. We have chosen the contour of integration along the real axis with an upward indentation at $\alpha=k$. The phase of $\beta$ is defined by

$$
p h \beta=0, \quad \alpha<k ; \quad p h \beta=-\pi / 2, \quad \alpha>k .
$$

With the help of the identity

$$
J_{1}(x) Y_{0}(x)-J_{0}(x) Y_{1}(x)=2 / x x,
$$

$I_{t}(z)$ may be broken into two parts

$$
I(z)=\frac{2 k a}{Z_{0}} \int_{0}^{\infty} \frac{H_{1}^{(2)}(\beta a) \cos \alpha z}{j \beta H_{0}^{(2)}(\beta a)} d \alpha
$$

and

$$
I_{i}(z)=\frac{2 k a}{Z_{0}} \int_{0}^{\infty} \frac{j J_{1}(\beta a) \cos \alpha z}{\beta J_{0}(\beta a)} d \alpha .
$$

The first of these expressions gives the current distribution on the outer surface of the tubular model. Current on the inner surface is described by (14). Only the exterior solution is of interest in antenna theory. Incidentally, (13) is in agreement with the work of Silver and Saunders [1950] who solved the exterior boundary value problem of an infinite cylinder with a window of arbitrary size and shape in which the electric field is known. Equation (13) follows from their work if the window is allowed to become a narrow circumferential gap and the usual boundary condition relating surface current density and magnetic field is imposed.

The task now at hand is to integrate (13) in such a way that $I(z)$ may be examined arbitrarily close to $z=0$. It is helpful to break the integrand into real and imaginary parts and write

$$
J(z)=\int_{0}^{\infty}[R(\alpha)+j W(\alpha)] \cos \alpha z d \alpha,
$$

or

$$
I(z)=r(z)+j i(z),
$$


where

$$
\begin{array}{lll}
R(\alpha)=\frac{4 k}{\pi Z_{0}} \frac{1}{\beta^{2}\left[J_{0}^{2}(\beta a)+Y_{0}^{2}(\beta a)\right]}, & & \alpha<k ; \\
R(\alpha)=0, & & \alpha>k ; \\
W(\alpha)=-\frac{2 k a}{Z_{0} \beta}\left[\frac{J_{1} J_{0}+Y_{1} Y_{0}}{J_{0}^{2}+Y_{0}^{2}}\right]_{\beta a}, & \alpha<k ; \\
W(\alpha)=\frac{2 k a}{Z_{0}} \frac{K_{1}\left[\left(\alpha^{2}-k^{2}\right)^{1 / 2} a\right]}{\left(\alpha^{2}-k^{2}\right)^{1 / 2} K_{0}\left[\left(\alpha^{2}-k^{2}\right)^{1 / 2} a\right]}, & \alpha>k .
\end{array}
$$

Thus, the real part of $I(z)$ can be determined by an integration only on the finite range $0 \leq \alpha \leq k$. The singularity in $\dot{R}(\alpha)$ at $\alpha=k$ is integrable so that

$$
r(z)=\frac{4 k}{\pi Z_{0}} \int_{0}^{k} \frac{\cos \alpha z}{\beta^{2}\left[J_{0}^{2}(\beta a)+Y_{0}^{2}(\beta a)\right]} d \alpha .
$$

The combination of Bessel functions seen in (18) occurs so frequently in what follows that it is convenient to define

$$
D(x)=J_{0}^{2}(x)+Y_{0}^{2}(x) .
$$

Further reduction is accomplished by the change of variable, $\alpha=k \cos \theta$. We also let $k z=\zeta$ and $k a=K$. Then,

$$
r(\zeta)=\frac{4}{\pi Z_{0}} \int_{0}^{\pi / 2} \frac{\cos (\zeta \cos \theta)}{\sin \theta D(K \sin \theta)} d \theta .
$$

Now introduce the well-known identity

$$
\cos (\zeta \cos \theta)=\sum_{n=0}^{n=\infty} \epsilon_{n}(-1)^{n} J_{2 n}(\zeta) \cos (2 n \theta)
$$

$\boldsymbol{\epsilon}_{n}=1$ for $n=0$, and $\epsilon_{n}=2$ for $n>0$.

In terms of coefficients which can be obtained by numerical or approximate integration,

$$
r(\zeta)=\frac{4}{\pi Z_{0}} \sum_{n=0}^{n=\infty} R_{2 n} J_{2 n}(\zeta)
$$

with

$$
R_{2 n}=\epsilon_{n}(-1)^{n} \int_{0}^{\pi / 2} \frac{\cos (2 n \theta)}{\sin \theta D(K \sin \theta)} d \theta .
$$

Further discussion of the $R_{2 n}$ and a table of values can be found in the section entitled Numerical Analysis. It is sufficient at this point to note that only a few terms of (22) are required to provide an accurate representation of $r(\zeta)$ on the range of $0 \leq \zeta<2 \pi$. Since $I(z)$ is an even function, this corresponds to a full wavelength on either side of the feedpoint at $z=0$.

The determination of $i(z)$ is more difficult. By using the small argument formulas for the Bessel functions and modified Bessel functions which occur in (17) it can be verified that $W(\alpha)$ contains non- integrable singularities of opposite sign on either side of $\alpha=k$. A direct attack would involve evaluating

$$
i(z)=\int_{0}^{\infty} W(\alpha) \cos \alpha z d \alpha,
$$

where a standard notation has been used to denote the principal value of the integral. Defining $i(z)$ by means of the principal value of an improper integral is not hard to justify. The difficulty could have been avoided in the first place by detouring well away from $\alpha=k$ in defining the contour. Even with this admitted, a serious difficulty remains. $W(\alpha)$ is a complicated function and the integral must be performed numerically over a long range in $\alpha$ before $K_{1}$ and $K_{0}$ are accurately represented by their asymptotic behavior. To do this and maintain high accuracy for small $z$ is difficult, even with the aid of automatic computer facilities.

Fortunately, the difficulties inherent in using (24) can be avoided entirely. $R(\alpha)$ can be used to determine both $r(z)$ and $i(z)$. We rewrite (13) as

$$
I(z)=\int_{0}^{\infty} F(\alpha) \cos \alpha z d \alpha .
$$

An obvious substitution gives

$$
I(z)=-j \int_{0}^{\infty} F(\alpha) \sin \alpha z d \alpha+\int_{0}^{\infty} F(\alpha) e^{j \alpha z} d \alpha .
$$

Denoting the imaginary part of the first integral by $i_{1}(z)$,

$$
i_{1}(z)=-\int_{0}^{k} R(\alpha) \sin \alpha z d \alpha .
$$

A series form similar to (22) can be written using

$$
\sin (\zeta \cos \theta)=2 \sum_{n=0}^{n=\infty}(-1)^{n} J_{2 n+1}(\zeta) \cos (2 n+1) \theta
$$

One obtains

$$
\begin{aligned}
i_{1}(\zeta) & =\frac{4}{\pi Z_{0}} \sum_{n=0}^{n=\infty} I_{2 n+1} J_{2 n+1}(\zeta), \\
I_{2 n+1} & =(-1)^{n+1} 2 \int_{0}^{\pi / 2} \frac{\cos (2 n+1) \theta}{\sin \theta D(K \sin \theta)} d \theta .
\end{aligned}
$$

A table of $I_{1}$ and $I_{3}$ for various values of $K$ can be found in the next section.

There remains

$$
i_{2}(z)=\operatorname{Im} \int_{0}^{\infty} F(\alpha) e^{j \alpha z} d \alpha .
$$

We now choose a closed contour consisting of a line from the origin along the positive real axis with an upward indentation at $\alpha=k$, the infinite quarter circle from the real axis to the positive imaginary axis, and a return to the origin via the imaginary axis. If $z$ is positive the integral along the large quarter circle will vanish. There are no poles within the contour. Then 


$$
i_{2}(z)=\operatorname{Im} \int_{0}^{j \infty} F(\alpha) e^{j \alpha z} d \alpha .
$$

The substitutions $\alpha=j \eta, \gamma=\sqrt{k^{2}+\eta^{2}}$ reduce (31) to

$$
i_{2}(z)=\frac{4 k}{\pi Z_{0}} \int_{0}^{\infty} \frac{e^{-\eta z}}{\gamma^{2} D(\gamma a)} d \eta .
$$

Even $i_{2}(z)$ is determined by $R(\alpha)$. With the understanding that $R(j \alpha)$ is defined on an infinite range, whereas $R(\alpha)=0$ for $\alpha>k$, we can summarize in terms of cosine, sine, and Laplace transforms with $z$ as the transform parameter. Equations (18), (27), and (32) can be collected into

$$
\begin{aligned}
& \operatorname{Re} I(z)=\mathscr{F}_{c} R(\alpha), \\
& \operatorname{Im} I(z)=-\mathscr{F}_{s} R(\alpha)+\mathscr{L} R(j \alpha) .
\end{aligned}
$$

In this sense the real and imaginary parts of $I(z)$ are codetermined.

For computational purposes (29) and (32) are a considerable improvement over (24). However, (32) can be improved further. The first step is the trivial one of obtaining an integrand in terms of nondimensional quantities. If we let $\eta=k v$ and $k a=K$,

$$
i_{2}(\zeta)=\frac{4}{\pi Z_{0}} \int_{0}^{\infty} \frac{e^{-v \zeta}}{\left(1+v^{2}\right) D\left(K \sqrt{1+v^{2}}\right)} d v .
$$

With the substitution $u=K \sqrt{1+v^{2}}$ (34) is transformed to

$$
i_{2}(\zeta)=\frac{4 K}{\pi Z_{0}} \int_{K}^{\infty} \frac{e^{-(\zeta / K) \sqrt{u^{2}-K^{2}}}}{\left(\sqrt{u^{2}-K^{2}}\right) u D(u)} d u .
$$

The advantage of (35) over (34) is apparent from the asymptotic behavior of $[u D(u)]^{-1}$. At $u=10$ this function is approximated by $\pi / 2$ with an accuracy of 0.12 percent. Thus we need be concerned with numerical integration only on the range $K \leq u \leq 10$ no matter what the value of $K$. By contrast, the integrand of (34) cannot be replaced by its asymptotic behavior until $v \simeq 10 / K$. For large $K$ (35) can be integrated directly [Erdelyi et al., 1953] to give

$$
i_{2}(\zeta) \simeq \frac{\pi K}{Z_{0}}\left[\mathbf{H}_{0}(\zeta)-Y_{0}(\zeta)\right]
$$

where $\mathbf{H}_{0}(\zeta)$ is the Struve function of zeroth order.

A different strategy is to be employed for small $K$. To eliminate unnecessary repetition of constants during the derivation we define $K$ times the integral in $(35)$ as $f(\xi)$. We start with the identity

$$
\begin{aligned}
(\zeta) & =\frac{1}{D(K)} \int_{K}^{\infty} \frac{e^{-(\zeta / K) \sqrt{u^{2}-K^{2}}}}{\sqrt{u^{2}-K^{2}}} d u \\
& -K \int_{K}^{10}\left[\frac{1}{K D(K)}-\frac{1}{u D(u)}\right] \frac{e^{-(\zeta / K) \sqrt{u^{2}-K^{2}}}}{\sqrt{u^{2}-K^{2}}} d u \\
& -K \int_{10}^{\infty}\left[\frac{1}{K D(K)}-\frac{1}{u D(u)}\right] \frac{e^{-(\zeta / K) \sqrt{u^{2}-K^{2}}}}{\sqrt{u^{2}-K^{2}}} d u .
\end{aligned}
$$

The first of these is integrable. In the third integral we replace $[u D(u)]^{-1}$ by $\pi / 2$ and neglect $K$ in $\sqrt{u^{2}-K^{2}}$. The second term is defined as a special function, $q_{K}(\zeta)$, to be determined and tabulated by numerical integration. We now have

$$
\begin{aligned}
f(\zeta) \simeq \frac{\pi}{2 D(K)}\left[\mathbf{H}_{0}(\zeta)-Y_{0}(\zeta)\right]-q_{K}(\zeta) \\
+\left[\frac{1}{D(K)}-\frac{K \pi}{2}\right] \operatorname{Ei}(-10 \zeta / K)
\end{aligned}
$$

Finally,

$$
i(\zeta)=\frac{4}{\pi Z_{0}}\left[f(\zeta)+\sum_{n=0}^{n=\infty} I_{2 n+1} J_{2 n+1}(\zeta)\right]
$$

unless $K \geq 10$, in which case $\left(4 / \pi Z_{0}\right) f(\zeta)$ is replaced by (36).

\section{Numerical Analysis}

The coefficients $R_{2 n}$ and $I_{2 n+1}$ have in common a factor of the type

$$
T_{n}=\int_{0}^{\pi / 2} \frac{\cos n \theta}{\sin \theta D(K \sin \theta)} d \theta .
$$

Near $\theta=0$ the integrand of (40) becomes approximately

$$
\left\{\theta\left[1+\left(4 / \pi^{2}\right) \ln ^{2}(\gamma K \theta / 2)\right]\right\}^{-1},
$$

where $\ln \gamma=0.57722$. It is convenient to break the integration into two parts. In the first part $0<\theta<\epsilon$ and the integrand is replaced by the small argument formula (41). The singularity is integrable, a convenient substitution being $(2 / \pi) \ln (\gamma K \theta / 2)=y$. The result of integrating formally in the small $\theta$ region is

$$
\frac{\pi}{2}\left[\frac{\pi}{2}+\arctan \left(\frac{2}{\pi} \ln \frac{\gamma K \epsilon}{2}\right)\right] .
$$

Numerical integration was used in the remaining interval, $\epsilon<\theta<\pi / 2$, and the result added to (42). The parameter $\epsilon$ was always chosen so that the small argument formula for $\dot{Y}_{0}(K \sin \theta)$ was accurate to four significant figures. A short table of $R_{2 n}$ and $I_{2 n+1}$ coefficients, adequate for the computation of $I(\zeta)$ on the range $0<\zeta<1$, is presented in table 1 .

\begin{tabular}{l|c|c|c|c}
\multicolumn{5}{c}{ TABLE 1 } \\
\hline \hline \multicolumn{1}{c|}{$R_{0}$} & $I_{1}$ & $R_{2}$ & $I_{3}$ \\
\hline & & & & \\
0.01 & 0.5683 & -1.009 & -0.7759 & 0.6707 \\
.02 & .6678 & -1.168 & -.8631 & .7283 \\
.04 & .8054 & -1.383 & -.9694 & .7912 \\
.08 & 1.005 & -1.686 & -1.099 & .8550 \\
.15 & 1.277 & -2.086 & -1.244 & .9035 \\
& & & & \\
\hline
\end{tabular}

Tables of $q_{K}(\zeta)$, to be omitted bere for the sake of brevity, are being prepared for separate publication. However, a few of the technical details and a qualitative description of the results are of general interest. Recall that $q_{K}(\zeta)$ is defined by 
$q_{K}(\zeta)=K \int_{K}^{10}\left[\frac{1}{K D(K)}-\frac{1}{u D(u)}\right] \frac{e^{-(\zeta / K)} \sqrt{u^{2}-K^{2}}}{\sqrt{u^{2}-K^{2}}} d u$

The significant features of the design of $q_{K}(\zeta)$ are:

(a) For $K=10, q_{K}(\zeta)=0$. If $K \geq 10, q_{K}(\zeta)$ is not needed at all since the contribution to $i(\xi)$ from the imaginary axis integration is adequately represented by (36).

(b) The integrand defining $q_{K}(\zeta)$ is everywhere finite on a finite range of integration. This feature simplifies, to a great extent, the problems of accuracy control and computer programing.

A plot of a typical $q_{K}(\zeta)$, figure 1 , shows that this function is appreciable near the origin, concave upward, and highly damped.

The approximations introduced in the third term of (37) are not serious even for large $K$. Corrections can be derived by expanding the integrand in a series and integrating term by term. Setting $K=0$ in $\sqrt{u^{2}-K^{2}}$ simply provides the first term of the series. Correction terms turn out to be of the form $\zeta^{n} \mathrm{Ei}(-10 \zeta / K)$. Near $\zeta=0$ the correction is small. Near $\zeta=1$ the correction amounts to about 50 percent of the last term of (38) for $K=5$. The error decreases for smaller $K$. However, for $\zeta$ near $\zeta=1$ the contribution to the total current from the integral in question is small. The net effect is a small error in a large term near the origin passing over to a large error in a small term as $\zeta$ is increased. Fortuitously, the correction terms may be dropped entirely even when $\sqrt{u^{2}-K^{2}} \simeq u$ is a poor approximation.

The singularity in $i(\zeta)$ is expressed explicitly by $Y_{0}(\zeta)$ and $E i(-10 \xi / K)$. The graph of $i(\zeta)$ becomes infinite at $\zeta=0$, decreases rapidly in a short interval, and then changes in a small transition interval to a moderate slope. As $\zeta$ increases $i_{2}(\zeta)$ decreases and the Bessel functions in $i_{1}(\zeta)$ begin to dominate and establish the wave character of $i(\zeta)$. Plots of $i(\zeta)$ were made for various values of $K$. A typical plot for $K=0.01$ is shown in figure 2. The singularity in the current distribution does not appear in the theory of antennas of finite length as developed to date. One would like an analogous current distribution in the theory of the infinite antenna. We have followed the intuitive procedure of extrapolating from the slowly varying portion of $i(\zeta)$ into the region of small $\zeta$. The resulting graph with the rapidly varying portion of $i(\zeta)$ removed is designated as $i_{s}(\xi)$. The range of the singularity is arbitrarily defined by

$$
i\left(\zeta_{s}\right)=i_{s}(0) ; \quad z_{s} / \lambda=\zeta_{s} / 2 \pi
$$

For small $K, z_{s}$ is a small fraction of a wavelength. Values of $z_{s}$ estimated from graphs of $i(\zeta)$ for various values of $K$ are presented in table 2 . The first line for $K=0.001$ was estimated from the behavior of the remaining data. As $K$ is increased the solution turns very gradually into the logarithmic singularity. Figure 3 exhibits two extremes with plots of $i(\zeta)$ for

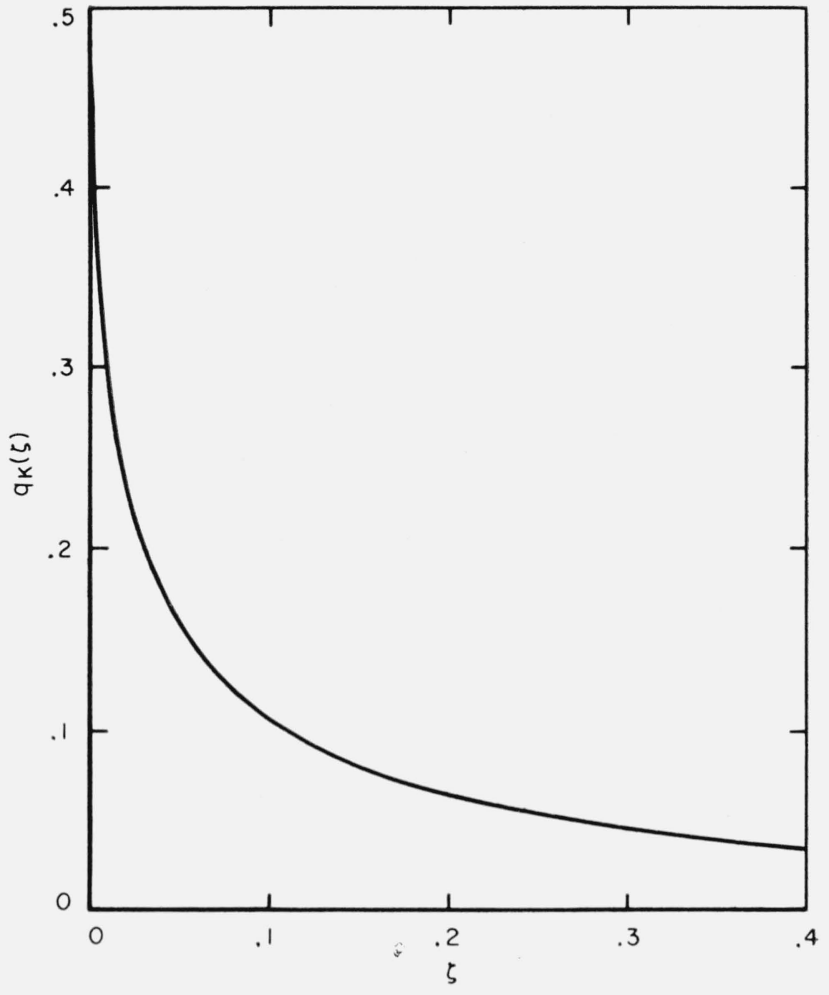

Figure 1. Graph of $q_{\mathrm{K}}(\zeta)$ versus $\zeta$ for $K=0.01$

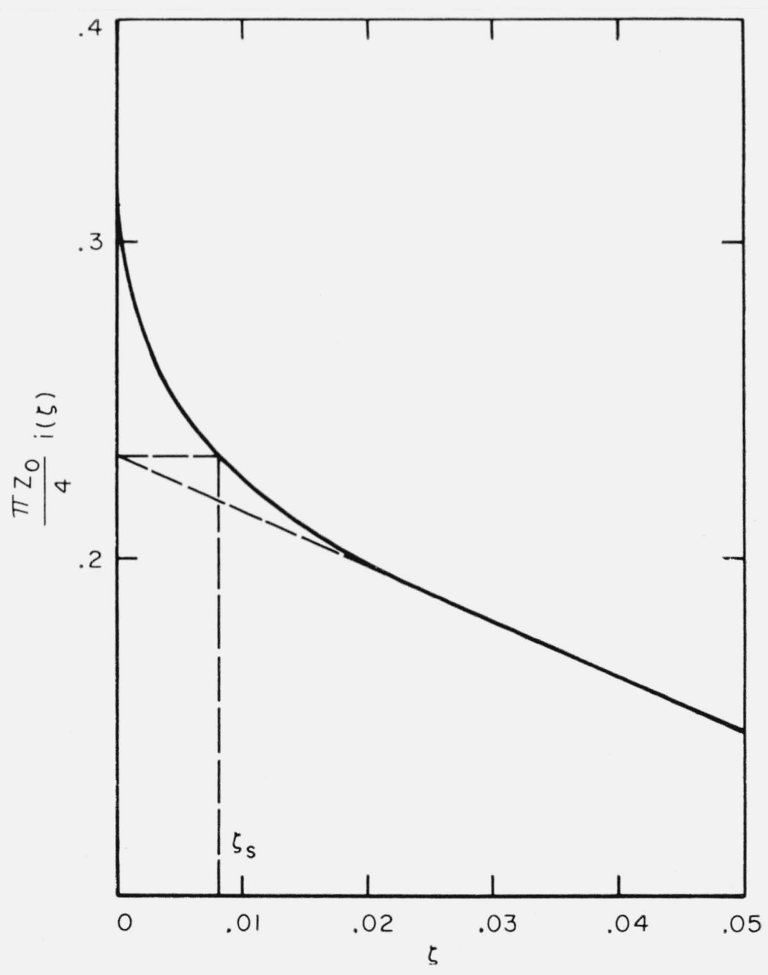

Figure 2. Expanded graph of $i(\zeta)$ versus $\zeta$ for $K=0.01$ 


\begin{tabular}{c|c|c}
\multicolumn{2}{c}{ TABLE 2} \\
\hline$K$ & $\zeta_{s}$ & $z_{s} / \lambda$ \\
\hline & & \\
\hline 0.001 & 0.006 & 0.00095 \\
.01 & .008 & .0013 \\
.02 & .010 & .0016 \\
.04 & .015 & .0024 \\
.08 & .020 & .0032 \\
.15 & .024 & .0038 \\
\hline
\end{tabular}

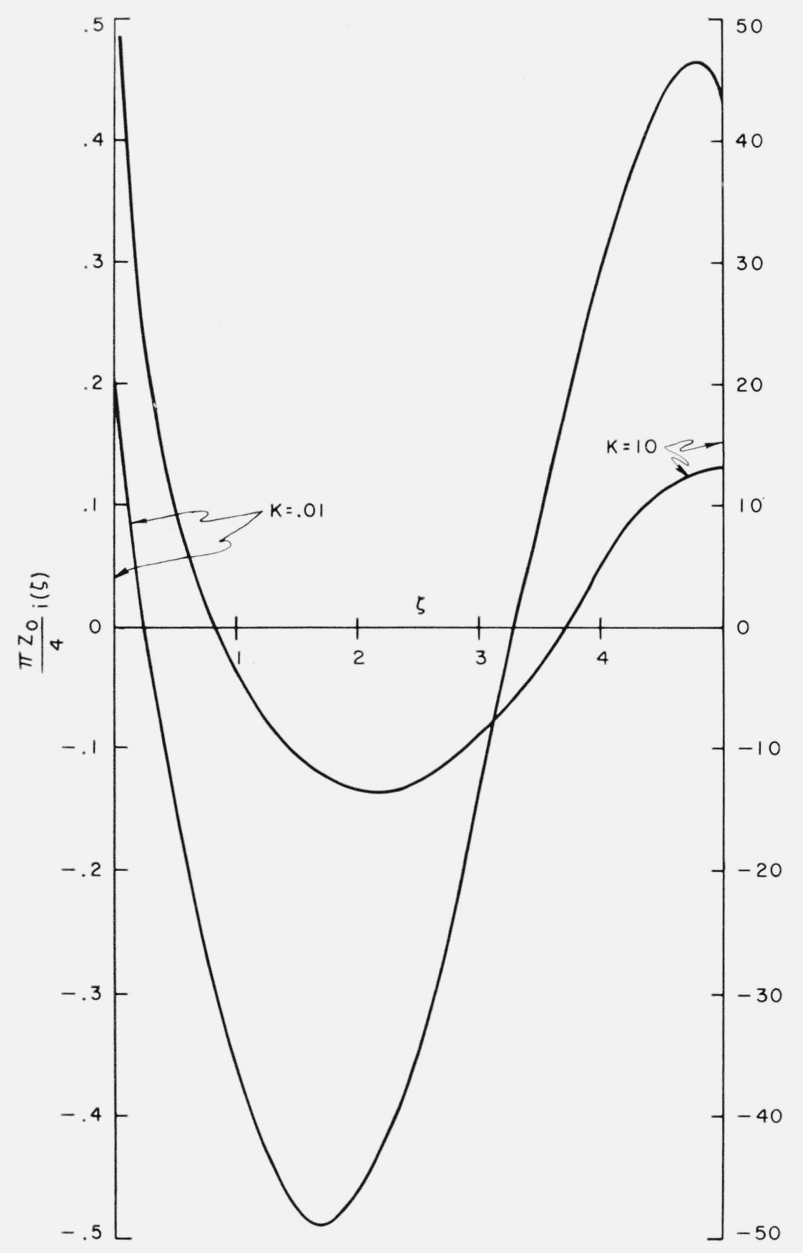

Figure 3. Graphs of $i(\xi)$ for $K=0.01$ and $K=10$

$K=0.01$ and $K=10$. The singularity in the graph for $K=0.01$ is not evident unless the scale of the abscissa is expanded considerably.

It is at once apparent that (8) is not an adequate estimate of the range of the singularity. The transition from moderate to large slope in the graph of $i(\xi)$ cannot be accounted for entirely by a simple logarithmic function. The qualitative conclusion that iterative and Fourier series solutions of feasible order will not detect the singularity remains intact.

\section{Modification for Nonzero Gap Width}

According to Silver and Saunders [1950] a gap in the region $-W \leq z \leq W$ is accounted for by a factor $(\sin \alpha W) / \alpha W$ in the integrand of (18). If this factor is included in the present work it will have very little effect on the functions $r(\zeta)$ and $i_{1}(\zeta)$. If the gap width is a small fraction of a wavelength $(\sin \alpha W) /$ $\alpha W$ will be nearly unity throughout the range of the integrals which define these functions. The appropriate modification turns out to be the inclusion of a factor (sinh $v w) / v w$ in the integrand of (34), giving

$$
i_{2}^{(w)}(\zeta)=\frac{4}{\pi Z_{0}} \int_{0}^{\infty} \frac{e^{-v \zeta}}{\left(1+v^{2}\right) D\left(K \sqrt{\left.1+v^{2}\right)}\right.} \frac{\sinh v w}{v w} d v,
$$

where $w=k W$. Denote the unmodified integrand by $F(v)$ for convenience. Then

$$
\begin{gathered}
i_{2}^{(w)}(\zeta)=\frac{2}{\pi Z_{0}} \int_{0}^{\infty} \frac{F(v)}{w v}\left[e^{-(\zeta-w) v}-e^{-(\zeta+w) v}\right] d v, \\
i_{2}^{(\delta)}(\zeta)=\frac{4}{\pi Z_{0}} \int_{0}^{\infty} F(v) e^{-v \zeta} d v
\end{gathered}
$$

where appropriate superscripts have been used to identify the separate cases. It is easy to show that

$$
i_{2}^{(w)}(\zeta)=\frac{1}{2 w} \int_{\zeta-w}^{\zeta+w} i_{2}^{(\delta)}(\zeta) d \zeta
$$

Thus, the required modification is a simple average over the evanescent part of the current distribution for a $\delta$-gap. Results for the two cases will be appreciably different only for small $\zeta$. It is more difficult to specify how this solution is to be matched to that for an appropriate transmission line and terminal zone structure. In thin antenna theory this difficulty is resolved by treating the antenna and terminal zone structure separately by suitable approximations and combining the results in an equivalent circuit. Clean separation of these two parts of the problem is not possible if the rapidly varying portion of $i_{2}^{(\delta)}(\zeta)$ extends over too large a region.

The simplicity of (48) can be attributed to the assumption of constant electric field in the gap. Wait [1959] has pointed out that a more realistic assumption leads to a factor of $J_{0}(\alpha w)$ in the integrand of (18) instead of $(\sin \alpha w) / \alpha w$.

\section{Impedance}

The conductance of the cylindrical antenna is easily defined for all values of $K$. It is given simply by

$$
G=r(0)=\frac{4}{\pi Z_{0}} \int_{0}^{\pi / 2} \frac{d \theta}{\sin \theta D(K \sin \theta)} .
$$

The integral is the definition of the coefficient $R_{0}$ defined earlier. If $K$ is large the function $D(K \sin \theta)$ takes on its asymptotic value of $(\pi K \sin \theta) / 2$ throughout most of the range of integration. Then the conductance-circumference ratio is 


$$
g=G / 2 \pi a \simeq \pi / Z_{0} \lambda .
$$

This is, as it should be, one-half the conductance per unit length of a slit in an infinite plane [Jordan, 1950]. Only one side of the cylindrical surface is counted because of the original splitting of the total current into antenna current and tube current. At $K=10$ (50) gives a value of 0.0835 mhos compared to a correct value of 0.0876 mhos. Cylinders with $K \geq 10$ are essentially infinite in diameter for the purpose of admittance calculations.

Susceptance is defined from the smoothed version of $i(\zeta)$ by

$$
B=\frac{4}{\pi Z_{0}} i_{s}(0)
$$

This represents an attempt to estimate, on a plausible basis, the value of susceptance observed in experimental work. Susceptances found in this manner are analogous to those calculated by the KingMiddleton theory or Fourier series solutions for the antenna of finite length. $A s \quad K$ is increased the introduction of a smoothed version of $i(\zeta)$ becomes less tenable. We have arbitrarily selected $K=0.15$ as that value above which the concepts of thin antenna theory are not to be applied. This is simply a matter of judgment based on the behavior of curves of $i(\zeta)$ versus $\zeta$ for various values of $K$.

For $K \geq 10$ a gap width, $2 W$, can be used in conjunction with (36) and (48) to define

$$
B=i^{(w)}(w)=\frac{1}{2 w} \int_{0}^{2 w} i^{(\delta)}(\zeta) d \zeta
$$

Only the Neumann function in (36) will contribute appreciably to (52). Passing to the small argument formula for $Y_{\mathrm{o}}(\zeta)$ and carrying out the indicated operations gives

$$
b=B / 2 \pi a \simeq \frac{2}{Z_{0} \lambda}\left(\ln \frac{\lambda}{2 \pi \gamma W}+1\right) .
$$

This is the susceptance per unit length for a slit in an infinite plane when the excitation is defined as a constant electric field in the slit. The slight difference between (53) and the corresponding formula in Jordan [1950] can be attributed to the differences in assumed excitation.

Table 3 gives the calculated admittances and impedances for small $K$.

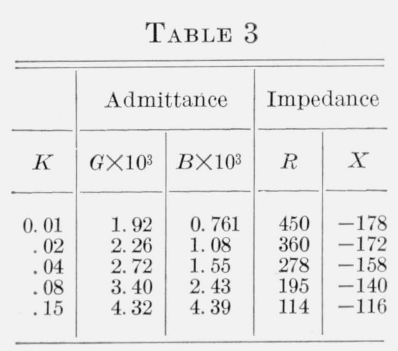

Altshuler [1961] recently reported the measured impedance of a traveling wave linear antenna with $K=0.04$ as $(320-j 110)$ ohms. The transmission line in his experiment was a coaxial line with a ratio of outer to inner diameters of the conductors of 8.13. The predicted value based on the present theory and appropriate terminal zone corrections is (346- $j$ 86) ohms.

\section{Conclusions}

The following classification of cylindrical antenna problems according to values of $K$, the antenna circumference in wavelengths, is suggested by the present theory and the literature. For $K<0.1$ a smoothed version of the exact solution of Hallén's integral equation can be used together with appropriate equivalent networks for realizable terminal zones to predict the behavior of physical antennas. Smoothing of the solution involves only a small region near the feedpoint of the idealized model to which the integral equation applies. The size of this region is of the order of a thousandth of a wavelength. This is much larger than had been previously supposed but still sufficiently small to justify the basic approach. Approximations to the kernel of the integral equation can be safely used for $K<0.02$. In particular, the King-Middleton theory is in agreement with high-order Fourier-series solutions using the exact kernel. For $0.1<K<10$ the antenna and terminal zone problems are not easily separated. A theory to provide both impedances and radiation characteristics for these diameters has not yet been developed. An adequate theory will have to involve a unified treatment of the terminal zone and a gap of nonzero width. Above $K>10$ the impedance properties of a long cylindrical antenna are well approximated by those for a slit in an infinite plane.

The theory and results presented here provide information about the singularity in theoretical antenna current. The calculated impedances should prove useful in the design of traveling-wave linear antennas.

The author thanks K. S. Kunz and C. W. Harrison, Jr. for continued encouragement and helpful discussions. J. F. Stanley and S. E. Dickson performed most of the calculations.

\section{References}

Altshuler, E. E., The traveling wave linear antenna, IRE Trans. on Ant. and Prop. AP-9, 4, 324-329 (1961).

Duncan, R. H., and F. A. Hinchey, Cylindrical antenna theory, J. Research NBS 64D, Radio Prop., No. 5, 569-584 $(1960)$.

Erdelyi, A., et al., Tables of integral transforms, Vol. I, 138 (McGraw-Hill Book Co., New York, N.Y., 1953).

Hallén, Erik, Theoretical investigations into the transmitting and receiving qualities of antennae, Nova Acta Regiae Soc. Sci. Upsaliensis, Ser. IV, 11, No. 4, 1-44 (1938). 
Hallén, Erik, Exact treatment of antenna current wave reflection at the end of a tube shaped cylindrical antenna, IRE Trans. on Ant. and Prop. AP-4, No. 3, 479 (1956).

Infeld, L., The influence of the width of the gap in the theory of antennas, Quart. Appl. Math. 5, 113 (1947).

Jordan, E. C., Electromagnetic waves and radiating systems, 579-583 (Prentice-Hall, Inc., New York, 1950).

King, R. W. P., The theory of linear antennas (Harvard University Press, Cambridge, Mass., 1956).
Silver, S., and W. K. Saunders, The external field produced by a slot in an infinite circular cylinder, J. Appl. Phys. 21, No. 2, 153-158 (1950).

Wait, J. R., Electromagnetic radiation from cylindrical structures (Pergamon Press, 1959).

$\mathrm{Wu}$, T., and R. W. P. King, Driving point and input admittance of linear antennas, J. Appl. Phys. 30, No. 1, 74, (1959).

(66D2-186) 